\title{
Iridium Ir 192
}

National Cancer Institute

\section{Source}

National Cancer Institute. Iridium Ir 192. NCI Thesaurus. Code C104270.

A radioactive isotope of iridium. Iridium-192 emits gamma rays and has a half-life of 74 days. A high dose rate of this radioisotope can be used in brachytherapy to treat tumors by selectively delivering a cytotoxic dose of radiation to the tumor site. 